\title{
Mathematical Approach to THD Optimisation in Multilevel Convertors
}

\author{
A. Jan Iwaszkiewicz ${ }^{1}$, B. Jacek Perz ${ }^{1}$ \\ ${ }^{1}$ The Electrotechnical Institute, Gdansk Branch, Poland \\ 80-557 Gdansk, Narwicka 1, Poland \\ phone:+48 58 3431291, fax+48 58 3431295, e-mail: A.jan.iwaszkiewicz@iel.gda.pl, B.jacek.perz@iel.gda.pl
}

\begin{abstract}
The paper is related to the problem of generating the high quality AC voltage waveforms using multilevel converters. This technique can be applied especially in the distributed power energy systems with renewable DC voltage sources like photovoltaic farms and fuel cells. A novel approach to the synthesis of AC voltage waveforms, considering minimising of THD by use of analytical methods like Fourier transform is presented. The analysis of the obtained waveforms is presented as well as examples of chosen parameter optimisation. The meaning of the THD factor from the construction point of view is analysed and a new bandwidth factor $\mathrm{THD}_{\mathrm{B}}$, containing more information about the frequency range of harmonic components is proposed in the paper.
\end{abstract}

\section{Key words}

THD, harmonics, multilevel converter, converter control, modulation strategy, power quality, waveform synthesis.

\section{Introduction}

There are many applications i.e. uninterruptible power sources or distributed power generation systems, where the demand is to generate $50 \mathrm{~Hz}$ sinusoidal voltage waveforms of relatively high quality. The voltage obtained from DC sources like storage accumulators, photovoltaic farms or fuel cells are converted to AC using power electronics converters. The quality of generated waveforms, especially THD (Total Harmonic Distortion) factor, should comply with the appropriate standards. The commonly used solution for this purpose is two level VSI (Voltage Source Inverter), controlled using PWM (Pulse Width Modulation) method. This method has well known disadvantages, related to high frequency switching, like power losses in switching elements and necessity of using special filters for high frequency components in the output voltages. These disadvantages can be reduced using multilevel converters and amplitude modulation method.

The mathematical approach to the control strategy of multilevel converters allows control of harmonic content and THD factor of output waveforms. The presented results have been obtained using Fourier transform waveform synthesis.

\section{Simple Fourier series waveforms synthesis}

Given is function $\varphi(\mathrm{x})$ :

$$
\varphi(x)=\left\{\begin{array}{lll}
1 & \text { for } & 0 \leq x<\alpha, \\
0 & \text { for } \text { other } x
\end{array}\right\} \quad \alpha \neq 0
$$

The scaling function $\varphi_{n}(x)$ is defined as following:

$$
\varphi_{n}(x)=\varphi(x-n \alpha) \text { for } n=\ldots,-2,-1,0,1,2, \ldots
$$

Scaling function represents square pulse of unitary amplitude and duration $\alpha$, The pulse position on $\mathrm{x}$ axis depends on parameter $n$. Some examples of the scaling functions are presented in Fig.1.

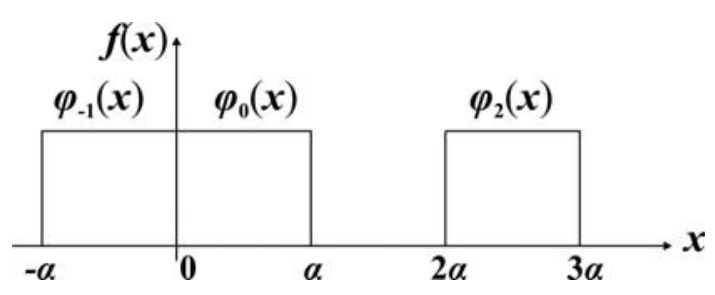

Fig. 1. Some examples of the scaling functions:

The transform of the function to generalized Fourier series based on the set of scaling functions $\left(\varphi_{\mathrm{n}}\right)$ :

$$
f(x)=\sum_{n=0}^{\infty} c_{n} \varphi_{n}(x) \text { where } c_{n}=\frac{\left(f, \varphi_{n}\right)}{\|\varphi\|^{2}}=\frac{\int_{a}^{b} f(x) \varphi_{n}(x) d x}{\alpha}
$$

Practically approximation consists in summation of finite number $\mathrm{N}$ of terms of the series. In power electronics applications the most important criterion of approximation accuracy of sinusoidal waveform is THD. An example of approximation of $f(\mathrm{x})=\sin \mathrm{x}$ function for $\mathrm{N}=6$ is presented in Fig. 2.

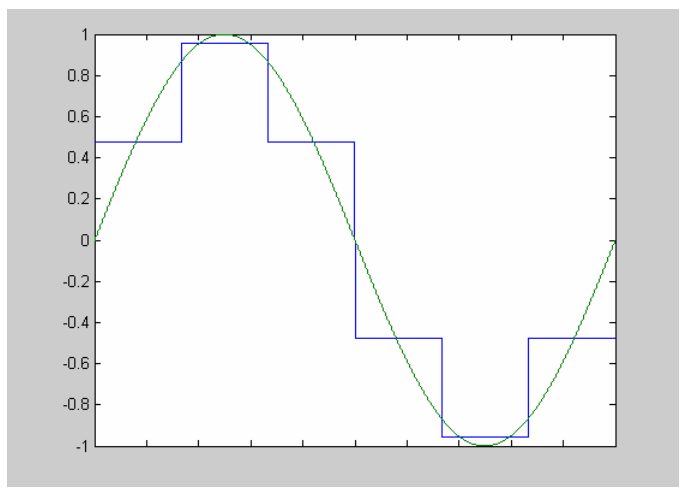

Fig. 2. The standard Fourier approximation of $f(x)=\sin x$ function for $\mathrm{N}=6$.

In Fig. 3 the spectrum analysis of this waveform is presented.

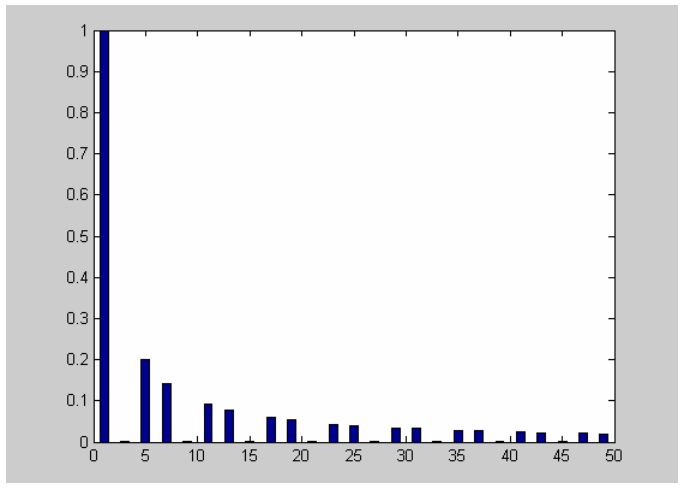

Fig. 3. The spectrum analysis of the waveform from Fig. 2. THD $=31.09 \%$. 
The results of Fourier approximation for consequent $\mathrm{N}$ are collected in Table 1.

Tab.1. Fourier approximation for different N.

\begin{tabular}{||c|c|c|c|}
\hline $\boldsymbol{F}_{\boldsymbol{N}}$ & $\alpha$ & $N_{\left|F_{N}\right|}$ & THD \\
\hline $\boldsymbol{F}_{N=2}$ & $\pi$ & 1 & $48.37 \%$ \\
\hline $\boldsymbol{F}_{N=6}$ & $\pi / 3$ & 2 & $31.09 \%$ \\
\hline $\boldsymbol{F}_{N=12}$ & $\pi / 6$ & 3 & $15.23 \%$ \\
\hline $\boldsymbol{F}_{N=16}$ & $\pi / 8$ & 4 & $11.41 \%$ \\
\hline $\boldsymbol{F}_{N=24}$ & $\pi / 12$ & 6 & $7.63 \%$ \\
\hline
\end{tabular}

The parameter $N_{\left|F_{N}\right|}$ denotes the number of demanded independent supply voltage sources.

\section{Optimised waveforms synthesis}

For the two step waveform shape the characteristic parameters are supply voltage ratio $\boldsymbol{V}_{\mathbf{0}} / \boldsymbol{V}_{\mathbf{1}}$ and the duration $\alpha$ of the first step. The duration of the second step is $\pi-2 \alpha$. For the waveform presented in Fig 2. these parameters are $\boldsymbol{V}_{\mathbf{0}} / \boldsymbol{V}_{\mathbf{1}}=0.5$ and $\boldsymbol{\alpha}=\boldsymbol{\pi} / 3$. The THD factor for this waveform is $31.09 \%$, but it is not the minimal possible THD value of two step waveform shape.

An example of approximation made using steps of different duration and amplitude is presented in Fig.4. The characteristic parameters for this waveform are: $\alpha=\pi / 6, V_{0}=0.2559, V_{1}=0.8270$.The spectrum analysis of this waveform is presented in Fig. 5.

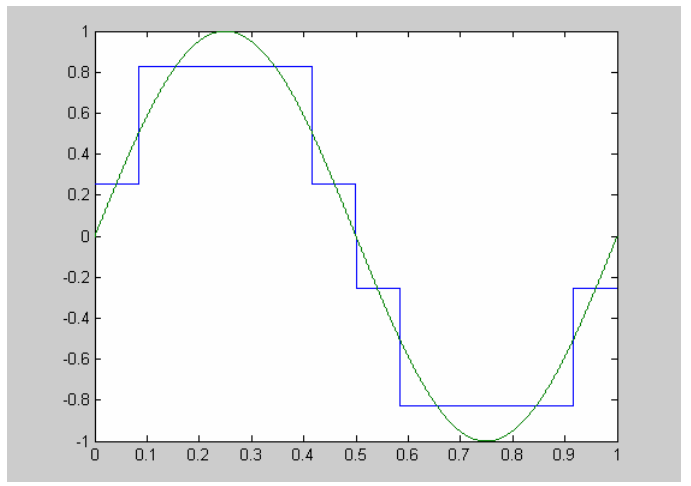

Fig. 4. The Fourier approximation of $f(\mathrm{x})=\sin \mathrm{x}$ function for $\alpha=\pi / 6, V_{0} / V_{1}=0.31$.

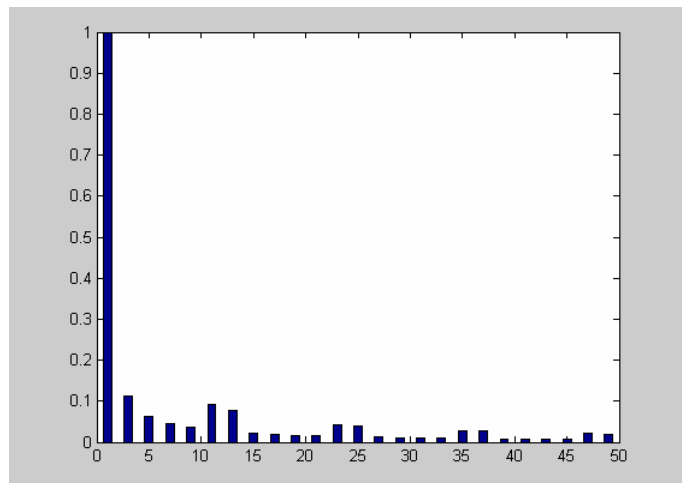

Fig. 5. The spectrum analysis of the waveform from Fig. 4. THD $=21.62 \%$.

THD factor of this waveform is much lower than obtained using simple Fourier synthesis, but contains low harmonic components including the third one.

It is possible to calculate the harmonic components of the two step waveform with characteristic parameters $V_{0}, V_{1}$ and $\alpha$.

$$
b_{k}=\frac{4}{k \pi}\left[V_{0}+\left(V_{1}-V_{0}\right) \cos (k \alpha)\right] \quad k=1,3,5, \ldots
$$

Using this relation it is possible to eliminate the chosen harmonic components. For example solving the appropriate set of equations it is possible to generate two step waveform without third and fifth harmonic components. The parameters of this waveform are: $\boldsymbol{V}_{\mathbf{0}}=0.3927$, $\boldsymbol{V}_{\mathbf{1}}=0.9481, \alpha=\pi / 4$. The resulting waveform is presented in Fig.6.

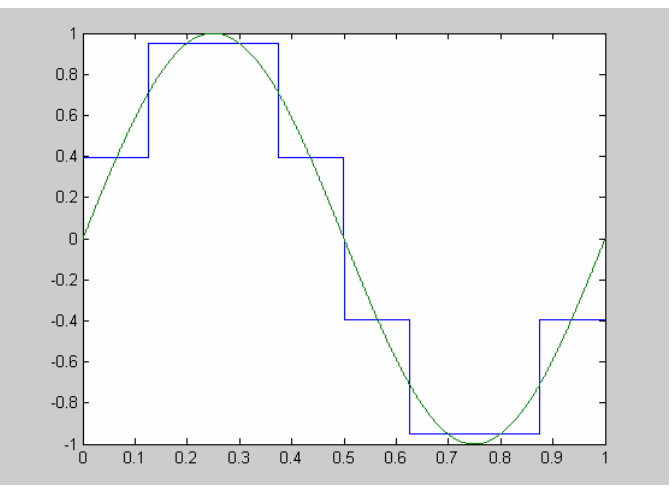

Fig. 6. The Fourier approximation of $f(\mathrm{x})=\sin \mathrm{x}$ function for $\alpha=\pi / 4, V_{0} / V_{1}=0.41$.

The spectrum analysis of this waveform is presented in Fig. 7 and shows absence of third and fifth harmonics.

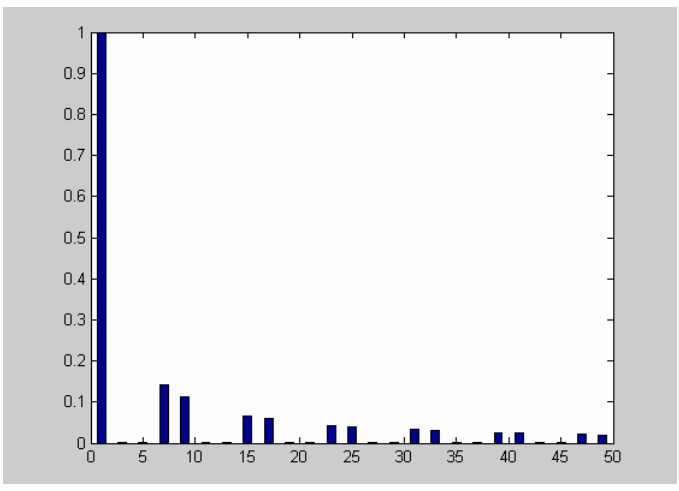

Fig. 7. The spectrum analysis of the waveform from Fig. 6. THD $=23.1 \%$.

By multivariable optimisation process it is possible to reduce the THD factor of the waveform down to the minimal value of $20.65 \%$. The parameters for this waveform are: $\boldsymbol{V}_{\mathbf{0}}=0.3139, \boldsymbol{V}_{\mathbf{1}}=0.8937, \boldsymbol{\alpha}=36^{\circ}$. The optimal waveform is presented in Fig. 8 and its spectrum analysis is presented in Fig. 9.

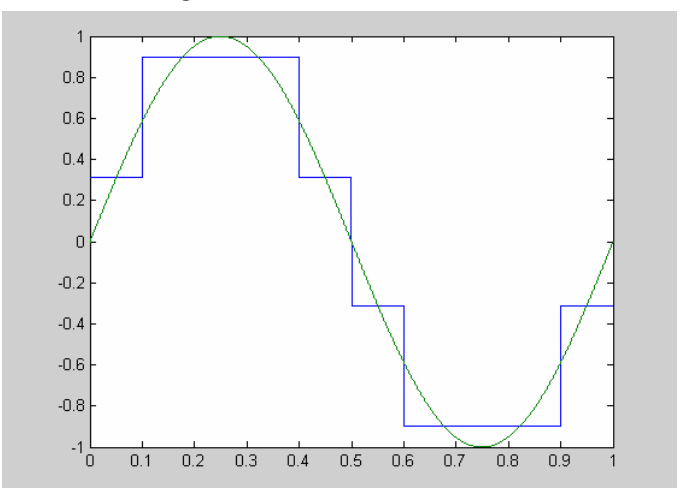

Fig. 8. The Fourier approximation of $f(\mathrm{x})=\sin \mathrm{x}$ function for $\mathrm{N}=6$, optimised for minimal THD. 


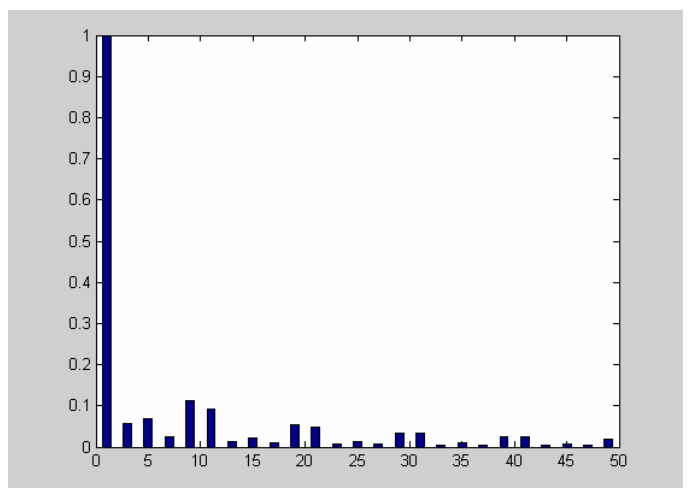

Fig. 9. The spectrum analysis of the waveform from Fig. 8. THD $=20.65 \%$.

\section{Bandwidth THD factor}

Simple comparison between results of spectrum analysis presented in Fig. 5 and Fig.7 shows that THD factor contains very little information about the shape of the harmonics spectrum, which is of crucial importance when additional filtration is necessary. The filter designed for the waveform from Fig 4. should have a bandwidth lower than $150 \mathrm{~Hz}$, but for the waveform from Fig. 6 the bandwidth should be $350 \mathrm{~Hz}$.

High power low-pass filter is rather huge and expensive device, which dimensions and price are increasing when the bandwidth decreases. So to compare the waveforms generated by different converters it is extremely important not only to know the total value of THD factor, but also the bandwidth of the harmonic components creating this value. For example it is much easier to construct the filter for the waveform from Fig. 6 than for the one from Fig. 4, though the THD of the former is higher than THD for the latter.

Considering this discrepancy in interpretation of the THD factor as a basis parameter for comparison the quality of sine-wave approximation generated by different equipment and using different control strategies, the new bandwidth $\mathbf{T H D}_{\mathbf{B}}$ factor has been defined and tested. The definition of this parameter is as follows:

$$
T H D_{B}=\frac{1}{b_{1}} \sqrt{\sum_{k=2}^{k=\infty}\left(\frac{b_{k}}{\log k}\right)^{2}}
$$

As a test for the $\mathbf{T H D}_{\mathbf{B}}$ factor the values of standard and bandwidth THD for the above mentioned waveforms have been calculated. The results are presented in Table 2 . Presented are THD values for the bandwidth limited to the 50 and 10 harmonic.

Tab.2. Comparison of THD and THD $_{\mathbf{B}}$ factors.

\begin{tabular}{||c|c||c|c||}
\hline \multicolumn{2}{||c|}{ Waveform from Fig. 4} & \multicolumn{2}{|c|}{ Waveform from Fig. 6 } \\
\hline THD $_{\mathbf{5 0}}$ & THD $_{\mathbf{5 0 B}}$ & THD $_{\mathbf{5 0}}$ & THD $_{\mathbf{5 0 B}}$ \\
\hline $20.63 \%$ & $28.93 \%$ & $22.00 \%$ & $22.63 \%$ \\
\hline THD $_{\mathbf{1 0}}$ & THD $_{\mathbf{1 0 B}}$ & THD $_{\mathbf{1 0}}$ & THD $_{\mathbf{1 0 B}}$ \\
\hline $14.30 \%$ & $26.41 \%$ & $18.10 \%$ & $20.50 \%$ \\
\hline
\end{tabular}

The values in the table shows that the waveform from Fig. 4 is better than the one from Fig. 6 when standard THD factor is considered, but considering $\mathbf{T H D}_{\mathbf{B}}$ leads to opposite conclusions.
In the $\mathbf{T H D}_{\mathbf{B}}$ definition each harmonic component has different scaling coefficient $\mathbf{w}_{\mathbf{k}}$, which is related to the number describing its position in harmonics spectrum in the following way:

$$
w_{k}\left(b_{k}\right)=\frac{1}{\log k}
$$

All harmonic components below tenth are scaled up while the components above this level are scaled down. The scale coefficients $\mathbf{w}_{\mathbf{k}}$ for selected harmonics are presented in Table 3.

Tab.3. Scaling coefficients $\mathbf{w}_{\mathbf{k}}$.

\begin{tabular}{|c|c||c|c||}
\hline $\mathbf{k}$ & $\mathbf{w}_{\mathbf{k}}$ & $\mathbf{k}$ & $\mathbf{w}_{\mathbf{k}}$ \\
\hline 2 & 3.32 & 70 & 0.54 \\
\hline 3 & 2.10 & 90 & 0.51 \\
\hline 4 & 1.66 & 100 & 0.50 \\
\hline 5 & 1.43 & 200 & 0.43 \\
\hline 6 & 1.28 & 300 & 0.40 \\
\hline 7 & 1.18 & 500 & 0.37 \\
\hline 8 & 1.11 & 1000 & 0.33 \\
\hline 9 & 1.05 & 2000 & 0.30 \\
\hline 10 & 1.00 & 3000 & 0.29 \\
\hline 30 & 0.68 & 5000 & 0.27 \\
\hline 50 & 0.59 & 10000 & 0.25 \\
\hline
\end{tabular}

The bandwidth $\mathbf{T H D}_{\mathbf{B}}$ factor contains important information about the harmonic components which are the most difficult for the filtering.

\section{Conclusions}

The proposed mathematical approach to the control strategy of multilevel converters allows significant reduction of THD. The presented example of three level (two step) converter proved the THD reduction from $31.09 \%$ to $20.63 \%$. It was also confirmed that by use the appropriate control strategy it is possible to eliminate chosen harmonic components (third and fifth in presented example). In high power applications this kind of converter's control strategy can be used to reduce the costs necessary to obtain the desired energy quality.

Observing that the value of standard THD factor can be misinterpreted because it does contain information about the shape of the harmonics spectrum, the new bandwidth $\mathbf{T H D}_{\mathbf{B}}$ factor has been defined. The presented examples proved that this new parameter contains important additional information related to the harmonics bandwidth. The $\mathbf{T H D}_{\mathbf{B}}$ factor can be used independently or together with the standard THD factor as a measure to compare the quality of waveforms generated by power electronics devices.

\section{References}

[1] Lida S., Masukawa S., and Kubota Y.: Improved Voltage Source Inverter With 18-Step Output Waveforms, IEEE Industry Applications Magazine, January/February 1998.

[2] Iwaszkiewicz J.: A Novel Recurrent Approach to the Output Voltage Filtering of the Inverter, EPQU 2003.

[3] Iwaszkiewicz J., Perz J.: Amplitude Modulation - an Alternative Method of Generating the Convertor Output Waveforms, ICREPQ Barcelona 2004

[4] Iwaszkiewicz J., Perz J.: Multilevel Converters for Distributed Power Generation Systems with DC Volage Sources, ICREPQ Saragossa 2005 